\title{
Virtual Reality as an Assistive Technology to Support the Cognitive Development of People With Intellectual and Multiple Disabilities
}

\author{
Rian Dutra da Cunha ${ }^{1}$, Rodrigo Luis de Souza da Silva ${ }^{1}$ \\ ${ }^{1}$ Departamento de Ciência da Computação - Universidade Federal de Juiz de Fora (UFJF) \\ 36036-900 - Juiz de Fora - MG - Brasil \\ eu@riandutra.com, rodrigoluis@gmail.com
}

\begin{abstract}
This study investigated the efficacy of a virtual reality-based cognitive and motor coordination training for people with intellectual and multiple disabilities, focusing on community participation. Ten people with disabilities were selected from an institutional care for people with disabilities and they were enrolled in a five-week experiment, which consisted in shopping in a virtual reality supermarket. The results show that the patients demonstrated statistically significant improvements regarding the task gave, also based on the observational analysis of the instructor and physiotherapist. Thus, the analysis indicates that the VR-based method can be effective in the cognitive development of those patients, improving their social inclusion and cognitive skills.
\end{abstract}

\section{Introduction}

A combination of traditional cognitive treatment and virtual reality-based methods can offer an effective, safe and interactive approach to the treatment of individuals with disabilities. Therefore, the current study discusses the contribution of virtual reality to promote social inclusion of people with intellectual and multiple disabilities, improving their community participation through a cognitive and motor skills training, which makes them more autonomous and able to do daily tasks.

The definition of social inclusion presented in [Simplican et al. 2015] refers to being accepted as an individual beyond disability, focusing on two domains - interpersonal relationships and community participation. In this context, community participation is the involvement in community activities that promote the development of interpersonal relationships, including leisure activities, political and civic activities or organizations, productive activities, like employment or education, religious and cultural activities and groups and consumption, or access to goods and services, which the current study is focused on.

Virtual Reality (VR) is increasingly being recognized as an effective tool for the rehabilitation of cognitive processes and functional abilities. The VR-cognitive rehabilitation framework was first proposed in [Rizzo and Buckwalter 1997], for children with attention-deficit activity disorder (ADHD). Traditional treatment requires specific exercises to improve the cognitive and motor skills through repetitive training. Intense repetition of tasks is necessary to reorganize the brain in a particular area of understanding; however, it requires immense demands both on the patient and the professional [Butler 2007]. In particular, because people with intellectual and multiple disabilities 
are difficult to engage, cognitive treatment approach applied to them can be a hard and complex task. Virtual reality-based methods, specially when it is game-like activities, are able to make cognitive and motor coordination treatment and rehabilitation more accessible to people with disabilities through their capacity to maintain their attention, providing an effective and safe training and educational media for those people.

In this paper, we verify the effectiveness of virtual reality as a tool to support the cognitive and motor coordination development of people with intellectual and multiple disabilities, focusing on the community participation, from a VR-based common daily task, monitoring the results and analyzing them from a quantitative and qualitative perspective. In Section 2, we describe the characteristics of the intellectual and multiple disabilities. In Section 3, we present related studies that investigated VR in the treatment of people with disabilities. In Section 4, we present the VR-DAD system developed in this study and its features. Section 5 describes our hypotheses, the details about this study as well as the procedure of the experiments. In Section 6 we present the results and in Section 7 our conclusions about this study.

\section{Intellectual and Multiple Disabilities}

Intellectual disability is a developmental disability characterized by limitations in intellectual functioning and adaptive behavior, resulting in the need for extraordinary supports for a person to participate in activities involved with typical human functioning [Schalock et al. 2010].

Another term related to intellectual disability is multiple disabilities, referring to people with severe disabilities, such as a sensory disability associated with a motor disability. Individuals with multiple disabilities usually presents more than one significant disability related to physical mobility, learning, speech, visual, hearing and brain injury. Besides, they can also exhibit sensory losses and behavior and/or social problems.

Therefore, intellectual disability is a state of functioning in which impairments to the central nervous system (CNS) (i.e., body functions and structures) result in activity limitations and participation restrictions, and, depending on the definition, a severe intellectual disability may be included in the term "multiple disabilities". Intellectual functioning is a type of human functioning, and is defined in the American Association on Intellectual and Developmental Disabilities (AAIDD) Manual on Definition, Classification and Support Systems in Mental Retardation [Schalock et al. 2010] as referring to a general mental ability that includes reasoning, planning, problem solving, abstract thinking, comprehending complex ideas, learning quickly and learning from experience.

\section{Related Studies}

Since computer technologies immersed into the society, many researches have been conducted to investigate the effectiveness of computer-based treatment of people with disabilities. Early studies, for instance [Colby 1973, Goldenberg 1979], suggested that using interactive computer software could encourage language use in children with disabilities. In 1973, Kenneth Mark Colby presented in [Colby 1973] a system which consisted of a television-like screen and a typewriter-like keyboard in front of which a child sits. In the experiment, children could interact pressing the keys and symbols appeared on the screen accompanied by sounds of human voices and common noises. V. Bernard-Opitz said in 
VI Congresso Brasileiro de Informática na Educação (CBIE 2017)

Anais do XXVIII Simpósio Brasileiro de Informática na Educação (SBIE 2017)

[Bernardopitz et al. 1989] children with Autism is more enthusiastic when working with computers than in a "regular toy situation".

In 1995, Melissa Salem Darrow presented in [Darrow 1995] a summary of VR applications developed for people with disabilities, and discussed about the positive predictions for VR as an assistive technology for people with sensory, physical and emotional impairments. Authors in [Cromby et al. 1996] said virtual environments are an effective and safe training and educational media for people with learning disabilities and suggests further researches to understand their full potentials. Also, they said that participants in a VR-based experiments can make as many mistakes as they wish without suffering the real or dangerous consequences of their errors, which is substantially important in this line of research.

The virtual dolphinarium presented in [Cai et al. 2013] allows children with ASD to act as dolphin trainers, focusing on improving their functional development learning and behavior. Authors concluded that VR is effective in the treatment of people with intellectual and multiple disabilities. In [Smith et al. 2014], the feasibility of a virtual reality job interview training was evaluated with adults with Autism. Authors conclude that it is an accessible strategy for improving community-based outcomes among people with ASD, and stated VR-based training may be a efficacious tool to improve job interview skills for those individuals.

A virtual reality for vocational rehabilitation system for people with disabilities is presented in [Bozgeyikli et al. 2014]. The authors said VR enables safe immersion of potential employees in a range of scenarios they may encounter before in a real job. Strickland at al [Strickland 1997] stated that virtual reality offers the potential to regulate an artificial computer environment to better match the expectations and needs of individuals with Autism, describing a study which concentrated on using VR as a learning aid with an immersive headset system.

There is a wide range of possibilities regarding the use of virtual reality to promote the community participation of people with intellectual disabilities, and the current article aims to present an effective and low-cost system to support the cognitive development of people with intellectual and multiple disabilities, training common daily tasks focused on the community participation.

\section{VR-DAD System}

The VR-DAD (Virtual Reality-Based Daily Activities Development) system uses immersive virtual reality to provides a virtual environment for teaching people with intellectual and multiple disabilities regarding to common daily activities, offering the possibility for individuals to improve their skills and to be more autonomous. The development of this system depended on other areas such as physiotherapy and pedagogy, and it was accompanied by specialists of these areas, as well as design and computer science. The prototype presented in this article, provides the following advantages over other systems (section $3)$ :

- Low cost. It is not required any desktop computer nor extra device, such as movement and depth sensors. It is functional from a mid-range smartphone and simple virtual reality glasses (e.g., Google Cardboard); 
VI Congresso Brasileiro de Informática na Educação (CBIE 2017)

Anais do XXVIII Simpósio Brasileiro de Informática na Educação (SBIE 2017)

- Hand-based manipulation using augmented reality in a virtual reality environment, providing a more realistic and intuitive interaction;

- Auto leveling system, which fits the difficulty level to each individual according to his or her performance;

- Results-Based Monitoring. Through an online dashboard, which can be accessed on any internet browser, it is possible to monitor the results according to the activities metrics.

\subsection{Results-Based Monitoring}

Through an online dashboard, which can be accessed on any internet browser, it is possible to monitor the results according to the activities metrics. As soon as each game is initialized, the feedback system starts to get relevant data regarding to the participant performance. Lastly, when the game ends, the result is sent to the online system, described in section 4.4. Thus, from the data collected by the system, specialists are able to analyze the cognitive and motor improvement of the patients and make more precise decisions regarding their treatment.

In the pilot project, participants should go shopping in a virtual supermarket (Section 5). Based on that task, the metrics proposed in this version of the VR-DAD system are: total quantity of products to be taken, quantity of product type, price to be paid, time spent, attempts to select products off the shopping list, attempts to select quantity of products higher than requested, failed product selection attempts, how many times participant gave more/less money and how many times the money selection was reset.

\subsection{Design and 3D Environment}

In this project, our purpose was not create a realistic scenario, which would use realtime illumination, rendering shadows and high resolution textures. Realistic graphics would require a robust processing and it would not be possible to play it on mid-range smartphones, which is important to maintain a low cost.

Although the scenario was not much realistic, the project was designed to provide a real-world experience, and the immersion and the hand-based interaction were capable to offer that sensation to the participants. The 3D modeling was made using the software Autodesk Maya 2017 and the textures are a mix of digital painting and real pictures, using the software Adobe Photoshop CC.

\subsection{Hand-Based Interaction}

One of the innovative resources which offer advantages over the systems presented in section 3 is the hand-based interaction. To provide a real-world experience, specially considering the cognitive and motor impairments of the participants, an interaction performed with their own hand (e.g., grab products, get money) is easier, more intuitive and effective.

The hand-based interaction is made by augmented reality into the virtual reality environment. The smartphone is placed in the VR head-mounted display and the system uses its camera to recognize the augmented reality marker, which is located over the hand of the participant (Figure 1). By the real-time recognition, the system replaces the marker with a virtual hand and the individual is capable to handle $3 \mathrm{D}$ objects in the VR scenario. 
VI Congresso Brasileiro de Informática na Educação (CBIE 2017)

Anais do XXVIII Simpósio Brasileiro de Informática na Educação (SBIE 2017)

For instance, if patients puts the hand over a product, it is outlined, and if he or she keeps the hand over it during three seconds, the product is selected (if the product is in the shopping list). Thus this specific task trains both the movement precision and the arm extension.

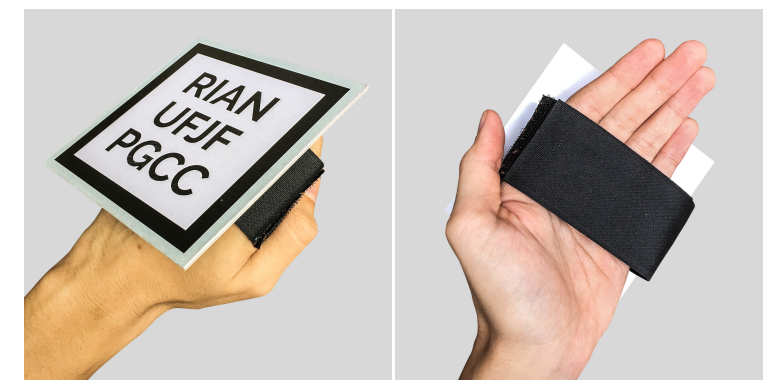

Figure 1. Augmented reality hand-based interaction

\subsection{Proof of Concept}

In the pilot project presented in this article, the virtual environment represents a small supermarket, where the participants have to go shopping (i.e., pick up the products and pay for them), which is fully described in Section 5. That kind of activity was commonly applied in the treatment of the patients in the institution where we selected the people with intellectual and multiple disabilities (section 5.3). The proof of concept was developed using the Unity 5.4 game engine, in which the Vuforia SDK was added. The Vuforia $S D K$ is an augmented reality software development kit for mobile devices that allows the creation of augmented reality applications. It uses computer vision technology to recognize image targets in real-time, and in this project it was used to track the hand of the participants, who used an augmented reality target attached to their hands (Figure 1).

\section{Materials and Methods}

\subsection{Research Questions}

The research questions of this study are: (1) How to teach day-to-day tasks to people with intellectual and multiple disabilities, making them more autonomous, regarding the common daily tasks? (2) How to use computation to develop assistive tools to improve cognitive and motor skills of people with intellectual disabilities?

Based on theses questions, the following hypotheses were formulated:

- Hypothesis 1 (H1): virtual reality can support the cognitive development, also improving the autonomous capabilities of people with intellectual and multiple disabilities;

- Hypothesis 2 (H2): hand-based interaction in a VR game-like activity can provide a more natural, attractive and intuitive interaction, thus being more effective both for the cognitive and the motor coordination development, instead of using a joystick or another device for the virtual objects manipulation.

\subsection{Experimental Design}

A single-subject design was used in the current study. Ten people with intellectual and multiple disabilities were enrolled in the study, which consisted of a training phase and a five-week follow-up session, with outcomes assessed throughout. 
VI Congresso Brasileiro de Informática na Educação (CBIE 2017)

Anais do XXVIII Simpósio Brasileiro de Informática na Educação (SBIE 2017)

\subsection{Participants}

For the case study, which was an illustrative case study done over five sections, 10 people with intellectual and multiple disabilities were selected from an institutional care for people with disabilities. They were selected under the ethical approval obtained from the ethics committee of the local university, as well as done with the consent of the responsible for the patients. The inclusion criteria were as follows: (1) diagnosis of multiple disabilities; (2) motor coordination non-severe, that is, patient should be able to use his or her hand to do the task; (3) basic cognitive capability, that is, individual had to be aware what was to be done in the task; (4) chronological age greater than 18.

\subsection{Equipment and Setting}

The experiments were conducted in the facilities of the institutional care for people with disabilities. The virtual reality application was tested in the smartphones Samsung Galaxy S3 (Android OS) and iPhone 6 Plus (iOS). During the sections, a Macbook Apple was used to mirror the smartphone screen, using an USB cable, thus the author and physiotherapist could see what the participant saw during the session. Regarding the virtual reality head-mounted display, a custom version of Beenoculus was used. Because the hand-based interaction is made by augmented reality (section 4.3), the HMD Beenoculus had to be customized with a frontal cut, right over the smartphone camera, so it is able to register the image and so create the augmented reality experience.

\subsection{Procedure}

In order to define an approach to daily activities teaching for people with intellectual and multiple disabilities using assistive technology, a case study has been defined. The case study consisted of a common daily task which involved go shopping in a supermarket, where patients should check, from a shopping list (e.g., Cookies x 2, Coffee x 3), what products they had to select from the supermarket shelves using their hands. The case study had the objective to identify the level of autonomy and understanding, and improve the performance of the patients to do common daily tasks. For example, how much time they spent to do the full task, how many mistakes they made when they were selecting the products and the quantity of each item, check if they understand the shopping list and if they could identify the supermarket sections where the products should be, as well as if they could pay the correct price of the purchase, selecting and giving the correct amount of money to the supermarket cashier. Figure 2 shows the virtual environment and the augmented reality hand-based interaction.

Before initial testing, the patients were provided with pilot experiment to ensure that they would understand the instructions associated to the VR task.

Among the 10 selected patients, one had major deficiency with balance. He could stand and walk without help from other people, however, after he used the VR headmounted display during about six minutes for the pilot task, he lost his balance when he took it out. After that experience, the patient and the physiotherapist decided not to do that task anymore. Another patient got a little dizzy when she took the VR-HMD out, but she enjoyed the experience and she did not get dizzy anymore, over the other sections. All other patients really enjoyed the virtual reality task and they always were excited to do it. Almost all the patients had difficulty in doing math (i.e., addition and subtraction), 
VI Congresso Brasileiro de Informática na Educação (CBIE 2017)

Anais do XXVIII Simpósio Brasileiro de Informática na Educação (SBIE 2017)

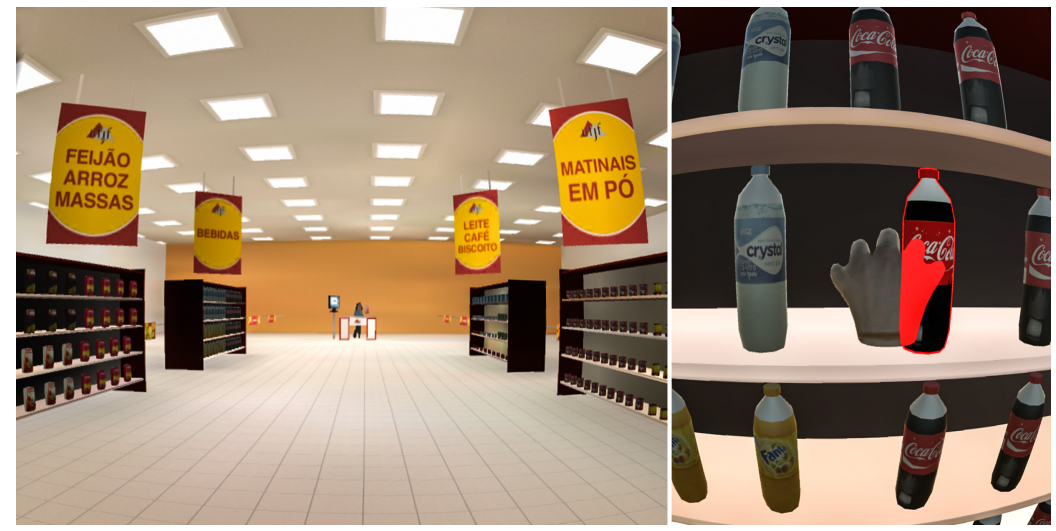

Figure 2. Virtual reality supermarket task and the augmented reality hand-based interaction.

thus, in a few times we had to help them at the moment of payment (i.e., select the money notes). Also, three of them could not read perfectly. In these cases, we had to read the shopping list and sections name for them, but they understood and identified clearly what were the products in the supermarket shelves.

\subsubsection{Five-Week Follow-up Session}

After the pilot experiment, a section per week was done with each one of the selected patients (Figure 3). The experiment was the same as the pilot task and the difficulty was increased at every task. For instance, for the latest tasks the patients were requested to pick up products located at the top and at the bottom of the shelves. Additionally, in each task the participants were more autonomous, that is, physiotherapist interfered less and less during the task. In the latest task, almost all the patients could shopping without any help.

At the end of the case study, the results were analyzed (section 6) to verify if there was an improvement regarding the patients' performance. Besides that, the physiotherapist gave her conclusion according to her observation during the period of the experiments. After the experiments, she observed an improvement related to balance and gross motor skills, in this case, specific to movement and coordination of the arms (section 6).

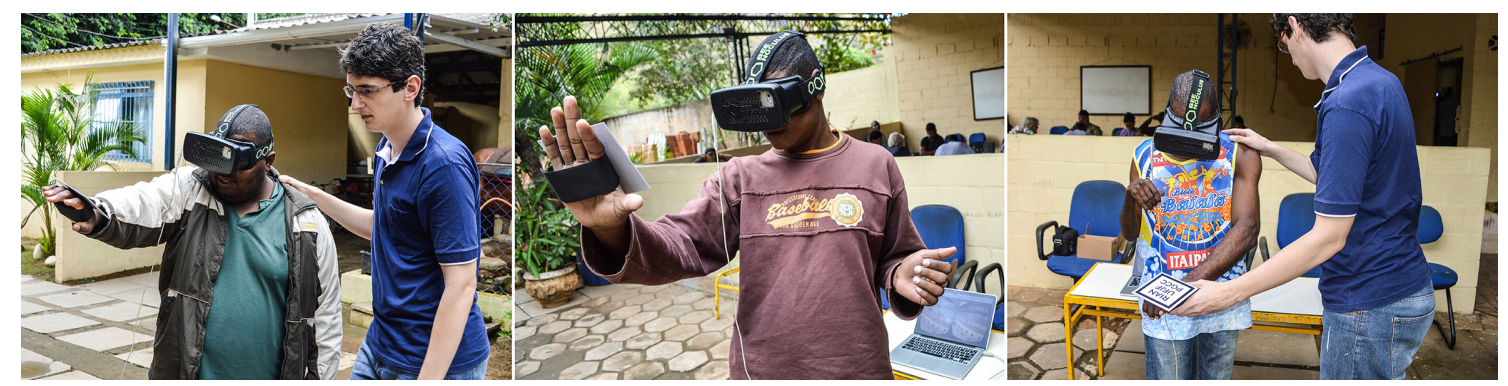

Figure 3. Some participants doing the task 
VI Congresso Brasileiro de Informática na Educação (CBIE 2017)

Anais do XXVIII Simpósio Brasileiro de Informática na Educação (SBIE 2017)

Table 1. Time spent result

\begin{tabular}{|l|c|c|c|c|c|}
\hline Game section & 1 (pilot) & 2 & 3 & 4 & 5 \\
\hline Mean & $5.996 \mathrm{~min}$ & $5.517 \mathrm{~min}$ & $3.860 \mathrm{~min}$ & $3.606 \mathrm{~min}$ & $2.429 \mathrm{~min}$ \\
\hline Standard deviation & 1.576 & 1.036 & 1.338 & 1.245 & 0.544 \\
\hline Margin of error & 0.864 & 0.568 & 0.734 & 0.683 & 0.298 \\
\hline
\end{tabular}

Table 2. Mistakes made during the task

\begin{tabular}{|l|c|c|c|c|c|}
\hline Game section & 1 (pilot) & 2 & 3 & 4 & 5 \\
\hline Mean & 6.286 & 6.444 & 1.444 & 1.111 & 0.889 \\
\hline Standard deviation & 2.812 & 4.096 & 1.333 & 1.691 & 1.054 \\
\hline Margin of error & 1.542 & 2.246 & 0.731 & 0.927 & 0.578 \\
\hline
\end{tabular}

\section{Data Analysis and Results}

After the five-week follow-up session, we analyzed the results sent to the online system by the VR application, stored at the end of each game. Overall, we noticed there was an improvement regarding the time spent and the mistakes made, indicating the virtual reality-based method was effective in this study.

In the first game session, the mean time spent by the selected patients was 5.996 minutes and after the intervention, they presented a mean time spent of 2.429 minutes, as can be verified in Table 1, which describes mean, standard deviation and margin of error using a confidence interval of $90 \%$ in order to make this study feasible.

During five weeks, the patients were submitted to tasks available by the VR-based game. Thus, at the end of each game session, each participant obtained his or her game session result provided by the game system. Table 2 shows the mean mistakes made over the five game sessions, as well as it presents standard deviation and margin of error using a confidence interval of $90 \%$. In order to make a more visible analysis, all mistakes were merged and counted, which are: attempts to select products off the shopping list, attempts to select quantity of products higher than requested, failed product selection attempts, how many times participant gave more/less money and how many times the money selection was reset.

Based on the results, it is possible to notice the participants spent less and less time to do the tasks and made less mistakes, showing an improvement on their performance. They could understand more clearly and faster what products they had to pick up from the shopping list according to the supermarket sections and then pay for them, showing us they were more autonomous after the training.

\subsection{Physiotherapist's feedback}

All the nine patients had their performance improved after the experiments, both in cognitive capabilities and in motor coordination. Some had greater improvement than others. The experiment was focused on the capability of the participants to go shopping on a supermarket, as autonomous as possible. Therefore, in addition to verifying an improvement in the results of the tasks (section 6), the physiotherapist and the instructor of the institution said there was an improvement related to their cognitive capabilities, such as logical reasoning and decision-making on common daily tasks, as well as doing math, 
VI Congresso Brasileiro de Informática na Educação (CBIE 2017)

Anais do XXVIII Simpósio Brasileiro de Informática na Educação (SBIE 2017)

even we had to help the patients in a few times. Thus, based on this study case, the (H1) was ratified, proving virtual reality has real potential to support the treatment of people with intellectual and multiple disabilities. Regarding the $(\mathrm{H} 2)$, related to the motor coordination improvement, we based on the observational analysis of the professionals, who noticed a small improvement on their gross motor skills, such as arm extension and more precision movements. The results related to the mistakes made also show they were more precise in the latest game sessions. Also, over the tasks, sometimes the patients were requested to pick up products located at the top and at the bottom of the shelves, which is more difficult. Thus, we could observe they perform these tasks easier.

\section{Conclusions and Future Works}

The results of the experiment presented in this paper demonstrated the effectiveness and feasibility of using virtual reality technologies for the cognitive and motor coordination training of people with intellectual and multiple disabilities. The low-cost intuitive handbased manipulation made the task more natural and easier to use, letting the participants more autonomous into the virtual environment, acting like in real world. Once the participants didn't have to learn how to use any devices (i.e., joysticks), they were able to focus on the provided task, indeed.

Overall, after the five-week follow-up session, the participants were able to do the task $40 \%$ faster on average, which represents an effective improvement, considering the short period, demonstrating improvement of the overall cognitive functioning of individuals with intellectual and multiple disabilities. Even in each session the task was not the same before (i.e., the shopping list changed every session, asking to grab different products in different locations), we noticed they were more confident and autonomous doing the that. The improvement may be attributed to exercising attentional processes during the engagement of the virtual reality training. The immersive and interactive nature of the VR-based method might offer an pleasurable experience for the participants, motivating them to further engage in the provided task.

In future versions of the VR-DAD system, it will be able to provide automatic evaluation according to the data collected. Thus, automatically VR-DAD would analyze the patients' performance and give us a feedback based on the scores, also it would predict the results based on the latest sessions, giving the pedagogue and the physiotherapist a more precise direction for the cognitive and motor coordination training of a patient; for instance, it would require a more complex shopping list to improve the patient's cognitive capabilities or it would direct activities that exercise the extension of the patient's arms if it is required for him or her.

The current study was not only about a simple task such as shopping in a supermarket, the objective was to present a pilot of a virtual reality-based task system which effectively support the cognitive and motor skills development of people with intellectual and multiple disabilities, focusing on their community participation, thus their social inclusion. Lastly, based on the analysis in Section 6 it was possible to prove that virtual reality-based method can be effective in the cognitive and motor skills development of people with intellectual disabilities. 
VI Congresso Brasileiro de Informática na Educação (CBIE 2017)

Anais do XXVIII Simpósio Brasileiro de Informática na Educação (SBIE 2017)

\section{References}

Bernardopitz, V., Roos, K., and Blesch, G. (1989). Computer-assisted instruction in autistic-children. Zeitschrift fur Kinder-Und Jugenpsychiatrie und Psychotherapie, 17(3):125-130.

Bozgeyikli, L., Bozgeyikli, E., Clevenger, M., Gong, S., Raij, A., Alqasemi, R., Sundarrao, S., and Dubey, R. (2014). Vr4vr: Towards vocational rehabilitation of individuals with disabilities in immersive virtual reality environments. In Virtual and Augmented Assistive Technology (VAAT), 2014 2nd Workshop on, pages 29-34. IEEE.

Butler, R. W. (2007). Cognitive rehabilitation. In Hunter, S. J. and Donders, J., editors, Pediatric Neuropsychological Intervention, page 444-464. Cambridge University Press.

Cai, Y., Chia, N. K., Thalmann, D., Kee, N. K., Zheng, J., and Thalmann, N. M. (2013). Design and development of a virtual dolphinarium for children with autism. IEEE transactions on neural systems and rehabilitation engineering, 21(2):208-217.

Colby, K. M. (1973). The rationale for computer-based treatment of language difficulties in nonspeaking autistic children. Journal of Autism and Developmental Disorders, 3(3):254-260.

Cromby, J., Standen, P. J., and Brown, D. J. (1996). The potentials of virtual environments in the education and training of people with learning disabilities. Journal of Intellectual Disability Research, 40(6):489-501.

Darrow, M. (1995). Virtual reality's increasing potential for meeting needs of persons with disabilities: What about cognitive impairments. Proceedings of the Third International Conference on Virtual Reality and Persons with Disabilities.

Goldenberg, E. P. (1979). Special technology for special children. Baltimore: University Park Press.

Rizzo, A. A. and Buckwalter, J. G. (1997). Virtual reality and cognitive assessment. Virtual Reality in Neuro-Psycho-Physiology: Cognitive, Clinical and Methodological Issues in Assessment and Rehabilitation, 44:123.

Schalock, R. L., Borthwick-Duffy, S. A., Bradley, V. J., Buntinx, W. H., Coulter, D. L., Craig, E. M., Gomez, S. C., Lachapelle, Y., Luckasson, R., Reeve, A., et al. (2010). Intellectual disability: Definition, classification, and systems of supports. ERIC.

Simplican, S. C., Leader, G., Kosciulek, J., and Leahy, M. (2015). Defining social inclusion of people with intellectual and developmental disabilities: An ecological model of social networks and community participation. Research in developmental disabilities, $38: 18-29$.

Smith, M. J., Ginger, E. J., Wright, K., Wright, M. A., Taylor, J. L., Humm, L. B., Olsen, D. E., Bell, M. D., and Fleming, M. F. (2014). Virtual reality job interview training in adults with autism spectrum disorder. Journal of Autism and Developmental Disorders, 44(10):2450-2463.

Strickland, D. (1997). Virtual reality for the treatment of autism. Studies in health technology and informatics, pages 81-86. 